\title{
An affordable computer-aided method for conducting Morris water maze testing
}

\author{
ANTHONY C. SANTUCCI \\ Manhattanville College, Purchase, New York
}

\begin{abstract}
A relatively inexpensive and reliable computer-aided method for assessing Morris water maze performance is described. The procedure uses SigmaScan digitizing software (Jandel Scientific, San Rafael, CA) to collect distance swum data, while escape latencies are measured with a hand-held stopwatch. Swim paths are videotaped for subsequent viewing and saved on disk as data sets of $x, y$ coordinates. At a later time, $x, y$ data files are printed and analyzed to obtain additional performance measures (e.g., distance swum in previous reinforced quadrant, heading angle, etc.). Intra- and interrater reliabilities associated with this method are presented. Correlation coefficients in the $r=$ $.85-.90$ and above range are reported for both reliability types. Given its approximate cost of $\$ 1,500$ (excluding the price of an IBM-compatible computer), the present procedure potentially can aid in performing instructional and research activities at small colleges which typically lack extensive equipment budgets.
\end{abstract}

Since 1981, when Morris presented his new method of assessing spatial learning and memory in rodents, the Morris water maze has been employed in many studies. Although there exist variations, this task's general method requires animals to find an escape platform located just beneath the water surface in a circular pool filled with opaque-colored water. The animal must use spatially distinct extramaze cues to solve the task successfully. The fact that Morris's method requires no pretraining and does not employ electric footshock or appetitive deprivation states has undoubtedly contributed to its widespread use.

Recently, a number of commercial companies have developed rather sophisticated computerized video tracking systems for IBM-compatible computers that allow researchers to automate Morris water maze data collection. A computer-aided procedure designed to run on a Macintosh Plus computer has also been described (Denenberg, Talgo, Waters, \& Kenner, 1990). Most systems not only record the time required by animals to reach the escape platform (escape latency, the primary dependent variable), but also assess a number of other measures such as time spent in reinforced and nonreinforced quadrants and annuli, heading angle, and distance swum before reaching the escape platform. Collected data are stored on disk, thus making them readily available for subsequent analysis and examination. Indeed the use of a computerized video tracking system is highly desir-

The author is in no way affiliated with Fibertech Engineering, Integrated Information Technology, Jandel Scientific, or Inset Systems. The author thanks the three referees of the manuscript for providing thorough, thoughtful and insightful reviews. Reprints of this article, or questions concerning its content, should be directed to A. C. Santucci, Department of Psychology, Manhattanville College, 2900 Purchase St., Purchase, NY 10577 (e-mail: santucci@mville.edu). able. Not only do these systems dramatically improve the efficiency with which data are collected, but their flexibility accommodates a variety of research and instructional paradigms. However, the rather steep price of such systems (from approximately $\$ 8,000$ to $\$ 18,000$ ) makes them prohibitively expensive, especially for small, liberal arts institutions that typically lack an extensive research and/or instructional equipment budget.

\section{General Description of Computerized Method}

In an attempt to glean some of the benefits of computerized video tracking without purchasing such a system, a relatively inexpensive procedure utilizing a standard video camera and digitizing software has been developed. Although the setup described here is not nearly as sophisticated or flexible as the commercially available tracking systems, it does ease and semiautomate some of the labor-intensive activities that are involved in Morris water maze testing. More importantly, the present system enables the experimenter to collect certain data that would otherwise be unobtainable.

The hardware setup necessary for this procedure is simple. A tripod-mounted video camera, connected to a standard portable television, is directed at a mirror suspended from the ceiling over the center of the black circular fiberglass pool (Fibertech Engineering, El Cajon, CA) used for testing (pool size: $121.1 \mathrm{~cm}$ in diameter, $68.6 \mathrm{~cm}$ high). The pool is filled with $25^{\circ}-26^{\circ} \mathrm{C}$ water, and approximately $300 \mathrm{~g}$ of tempera black nontoxic powdered paint are added to make the water opaque. A 26.7 $\mathrm{cm}$-high platform is submerged $1.3 \mathrm{~cm}$ below the surface of the water. Animals (rats or mice) are placed in the pool facing the wall at one of four start locations. After placing the animal in the pool, the experimenter sits in front of the television and computer, which are out of the animal's view. (Two people can be used to test an- 
imals; see Procedural Options and Other Applications below.)

As the experimenter measures escape latency with a hand-held stopwatch, she/he draws the swim path of the animal on an opaque digitizing tablet (Jandel Scientific, Model 2210) connected to an IBM-compatible computer. To aid in producing reliable digitized swim paths, templates of the pool with the four start locations and quadrant boundaries drawn on thin clear pieces of plastic are overlaid and affixed onto the digitizing tablet and television screen. With the use of a commercially available digitizing software package (SigmaScan, Version 3.90, Jandel Scientific, San Rafael, CA), a screen-drawn image is produced as the animal's path is tracked on the tablet. When the trial is completed, the software calculates the swim path distance (in centimeters), reports it on screen, and places it in the data worksheet. Swim speed (in centimeters/second) can be calculated by dividing the distance swum to the platform by the latency to the platform, to estimate motor and motivational competence. This measure can be performed automatically within the software's data worksheet, which is capable of applying any userdefined transformation to the collected data. Swim speeds are extremely helpful when one is trying to dissociate cognitive from noncognitive effects, especially in conducting aging, lesion, or pharmacology studies. Of course, since the digitizing tablet is only $12 \times 12$ in., the distance swum reported by the software must be corrected by multiplying it by the number of times the pool is larger than the digitizing tablet in order to obtain actual distance swum measurements. This transformation can also be performed automatically via the data worksheet module.

\section{Storage of Data}

Although latency and distance swum data are readily available and can be recorded easily at the end of each trial, it is often desirable to obtain additional performance measures. However, given the temporal constraints of conducting an experiment, it is often impossible to perform subsequent detailed performance analyses during individual test sessions. Fortunately, the present method allows the experimenter to save the performances of individual animals for later study. Storage can be performed in one of four ways. First, since most computers and monitors are capable of printing the screen-drawn image via the PRINT SCREEN keyboard command used in conjunction with the GRAPHICS.COM DOS utility, the screen-drawn path of each animal can be printed immediately after each animal's test session and studied later. However, depending on the particular hardware used, this may take up to several minutes per output. Alternatively and preferably, SigmaScan can be calibrated to save images of individual swim paths on disk as data files of $x, y$ coordinates. Saving $x, y$ coordinates is performed with a few keystrokes that require little additional time. Swim paths can be reproduced from these $x, y$ data sets and printed from within either SigmaScan (via PRINT SCREEN) or several spreadsheet/graphics ap- plications such as SigmaPlot, Jandel's scientific plotting program. As a third option, copies of screen-drawn swim paths can be obtained with a low-cost commercially available TSR screen grabber such as Hijack (Inset Systems, Brookfield, CT; approximately $\$ 50.00$ ). Lastly, a permanent record of maze performance can be created on videotape. Because videotaping provides an opportunity to acquire additional performance measures (e.g., time spent and distance swum in reinforced quadrant, etc.; see Additional Measurements below), its use in conjunction with one of the other storage options, preferably the $x, y$ storage option, is strongly encouraged.

\section{Experimental Results}

Two experiments were performed to assess the procedure's sensitivity, accuracy, and reliability. The first experiment assessed the sensitivity and accuracy of the procedure's ability to store $x, y$ data points by determining how many $x, y$ data points are sampled and stored per unit of swim time. Seventeen fictitious 30 -sec swim paths of varying distances $/$ speeds $(486-1,086 \mathrm{~cm} ; 16.2-36.2 \mathrm{~cm} / \mathrm{sec})$ were examined. A $286 / 8 \mathrm{MHz}$ IBM-compatible computer was used, with a compressed $20-\mathrm{Mb}$ hard drive and a CGA video. The wide range of swim distances/speeds was selected to ensure inclusion of the distances/speeds typically exhibited by rats. The number of $x, y$ coordinates sampled, the number of $x, y$ coordinates sampled/second, the number of $x, y$ coordinates sampled/centimeter of swimming, and centimeters of swimming/sampled coordinate are presented in Table 1.

As can be seen in this table, irrespective of the swim path's distance/speed, the present hardware setup allowed for the sampling of about $290 x, y$ coordinates. This translated into approximately 9.7 coordinates being sampled every second. Because the sampling rate was simply a function of time rather than a function of swim distance/speed, the number of coordinates sampled per centimeter of swimming decreased as the swim distance/speed increased. The number of coordinates sampled per centimeter of swimming ranged from 0.60 , a value associated with the shortest/slowest swim $(486 \mathrm{~cm}$; $16.2 \mathrm{~cm} / \mathrm{sec}$ ), to 0.27 , a value associated with the longest/ fastest swim $(1,086 \mathrm{~cm} ; 36.2 \mathrm{~cm} / \mathrm{sec})$. In other words, when the shortest swim path was digitized, one coordinate was sampled every $1.7 \mathrm{~cm}$ of swimming; when the longest swim path was analyzed, one coordinate was sampled every $3.7 \mathrm{~cm}$ of swimming. On the basis of the results from numerous prior studies, it is known that swim speeds for most rats range between 18 and $30 \mathrm{~cm} / \mathrm{sec}$. Therefore, the more usual state of affairs is for one coordinate to be sampled about every $2-3 \mathrm{~cm}$ of swimming. Sampling at this rate has proved to be very adequate for accurately reproducing swim path performances. It should be noted that since sampling rates are hardware dependent, higher sampling rates can be achieved when faster 386 or 486 machines are used. For example, pilot data indicated an approximate $40 \%$ increase in sampling rates when a $386 \mathrm{SX} / 16-\mathrm{MHz}$ system with an $80-\mathrm{Mb}$ compressed hard drive and a VGA monitor was used. 
Table 1

Data Associated With the Sampling of $x, y$ Coordinates Obtained From Seventeen 30-sec Trials During Which a Range of Fictitious Swim Path Distances Were Digitized

\begin{tabular}{cccccc}
\hline $\begin{array}{c}\text { Swim Distance } \\
(\mathrm{Cm})\end{array}$ & $\begin{array}{c}\text { Swim Speed } \\
(\mathrm{Cm} / \mathrm{Sec})\end{array}$ & Sampled & Sampled/Sec & $\begin{array}{c}\text { Sampled/Cm } \\
\text { of Swimming }\end{array}$ & $\begin{array}{c}\text { Cm of Swimming/ } \\
\text { Coordinate }\end{array}$ \\
\hline 486 & 16.2 & 290 & 9.7 & 0.60 & 1.7 \\
506 & 16.9 & 282 & 9.4 & 0.56 & 1.8 \\
518 & 17.3 & 287 & 9.6 & 0.55 & 1.8 \\
541 & 18.0 & 294 & 9.8 & 0.54 & 1.8 \\
593 & 19.8 & 288 & 9.6 & 0.49 & 2.1 \\
618 & 20.6 & 293 & 9.8 & 0.47 & 2.1 \\
621 & 20.7 & 291 & 9.7 & 0.47 & 2.5 \\
714 & 23.8 & 287 & 9.6 & 0.40 & 2.5 \\
744 & 24.8 & 294 & 9.8 & 0.40 & 2.7 \\
809 & 27.0 & 297 & 9.9 & 0.37 & 2.9 \\
817 & 27.2 & 288 & 9.6 & 0.35 & 2.9 \\
828 & 27.6 & 293 & 9.8 & 0.35 & 3.1 \\
848 & 28.3 & 291 & 9.7 & 0.34 & 3.4 \\
914 & 30.5 & 290 & 9.7 & 0.32 & 3.7 \\
985 & 32.8 & 288 & 9.6 & 0.29 & 0.29 \\
990 & 33.0 & 290 & 9.7 & 0.27 & \\
1086 & 36.2 & 293 & 9.8 & 0.8 & No. of $x, y$ Coordinates \\
\hline
\end{tabular}

Note--A 286/8 MHz IBM-compatible computer with a compressed 20-Mb hard drive and a CGA video was used to collect these data.

Experiment 2 assessed the reliability of the present procedure. Two raters, one experienced (Rater 1) and one inexperienced (Rater 2), digitized five actual videotaped swims of rats whose escape latencies ranged from 3 to $120 \mathrm{sec}$. Each swim was digitized 10 times, so that each rater provided 50 digitizations. The means and standard deviations for these measurements, in addition to intraand interrater reliability coefficients, are presented in Table 2.

Both raters generally were very internally consistent, typically exhibiting intrarater reliabilities of $r=.94$ and above (all $p s<.02$ ). There was, however, one exception. The inexperienced rater (Rater 2) was somewhat less consistent in judging the $3-\sec$ trial $(r=.72, p>.10)$. Even the experienced rater, despite yielding a statistically significant intrarater reliability coefficient, exhibited a fair amount of variability ( $S=9.9 \%$ of the mean) when assessing the 3-sec swim path. It should be realized, however, that even though the potential for substantial variability exists when one is digitizing short distances, the present method would still present no difficulty for discriminating between the distances of two relatively short paths. Swims, for example, requiring escape latencies of 3 and $10 \mathrm{sec}$ would still most likely be discernible, according to the digitized distances provided by even an inexperienced rater. ${ }^{1}$ With respect to interrater reliability, correlation coefficients ranging from $r=.85$ to .96 (all $p s<.01$ ) were obtained for the five videotaped trials. Together, these data support the view that the present method is reliable for assessing Morris maze performance.

\section{Additional Measurements}

Partial impetus for using sophisticated computerized video tracking systems is their ability to dispense de- tailed performance information. In addition to providing path length and escape latency data, many systems, as mentioned above, also report turning angles, time spent in reinforced and nonreinforced quadrants and annuli, dwell time spent in various areas of the maze, heading angle to the escape platform, and frequency of entry into various zones of the pool. These data are collected simultaneously and are summarized in concise, easily readable session reports. By reanalyzing saved data, the present procedure is capable of obtaining some of these additional measures. Admittedly, however, collecting additional measurements requires a fair amount of time and effort on the part of the experimenter. Nevertheless, by subsequently digitizing printed outputs, path distances in various regions of the pool, including distances swum in reinforced and nonreinforced quadrants and annuli, are obtainable. These particular measures are especially useful for analyzing performances of probe trials. Probe trials are those trials during which swims occur in the absence of the escape platform to determine an animal's preference for searching an area in which the escape platform has been located previously. Determining such preferences is very important for dissociating spatial from nonspatial strategies. In addition, by using the angle measurement function from within SigmaScan, the heading angle the animal takes en route to the platform also can be assessed. Finally, videotapes can be played back and analyzed to measure time spent in various areas of the maze (with a stopwatch) and to count the number of quadrant or region entries (performed manually or with SigmaScan's tally function).

\section{Procedural Options and Other Applications}

The present procedure can be modified in a number of ways. Even though the total cost is already relatively low, 
Table 2

Means, Standard Deviations (S), and Reliability Measures Associated With 2 Raters Digitizing 5 Videotaped Actual Swims of Varying Latencies

\begin{tabular}{|c|c|c|c|c|c|}
\hline & \multicolumn{5}{|c|}{ Escape Latencies } \\
\hline & $3 \mathrm{sec}$ & $21 \mathrm{sec}$ & $42 \mathrm{sec}$ & $79 \mathrm{sec}$ & $120 \mathrm{sec}$ \\
\hline \multicolumn{6}{|l|}{ Rater 1 (experienced rater) } \\
\hline Mean distance swum $(\mathrm{cm})$ & 62 & 550 & 1,006 & 1,871 & 3,330 \\
\hline$S$ & 6.1 & 28.3 & 17.6 & 39.6 & 56.3 \\
\hline$S \%$ of mean & $9.9 \%$ & $5.1 \%$ & $1.7 \%$ & $2.1 \%$ & $1.7 \%$ \\
\hline$r$ (split-half reliability) & .996 & .97 & .97 & .99 & .96 \\
\hline$p$ & $p<.001$ & $p<.01$ & $p<.01$ & $p<.01$ & $p<.01$ \\
\hline \multicolumn{6}{|l|}{ Rater 2 (inexperienced rater) } \\
\hline Mean distance swum $(\mathrm{cm})$ & 63 & 554 & 1,021 & 1,818 & 3,356 \\
\hline$S$ & 11.1 & 13.3 & 12.9 & 53.2 & 49.2 \\
\hline$S \%$ of mean & $17.6 \%$ & $2.4 \%$ & $1.3 \%$ & $2.9 \%$ & $1.5 \%$ \\
\hline$r$ (split-half reliability) & .72 & .998 & .96 & .99 & .94 \\
\hline$p$ & n.s. & $p<.001$ & $p<.01$ & $p<.01$ & $p<.02$ \\
\hline$r$ (interrater reliability) & .96 & .87 & .85 & .96 & .96 \\
\hline$p$ & $p<.001$ & $p<.01$ & $p<.01$ & $p<.001$ & $p<.001$ \\
\hline
\end{tabular}

Note-Each rater digitized each of the 5 swim paths 10 times.

expenses can be decreased further by eliminating the video camera, mirror, and television set. Such a setup would necessitate that the experimenter digitize the swim path while directly observing the animal in the pool. Of course, if this were done, sufficient precautions would have to be taken to minimize the potential disruptive effects posed by having the experimenter be seen by the animal. In addition, because she/he would serve as an extramaze cue, the experimenter would have to be certain to position herself/himself consistently in the same spatial location relative to the pool during each trial. Eliminating the video camera would obviously result in not having a permanent record of the actual swim and thus would prevent an opportunity to obtain some additional measurements. This, in my opinion, would represent a serious limitation.

Another option is to use a clear glass digitizing tablet rather than an opaque tablet. The glass tablet could be positioned on top of an upwardly tilted television screen, allowing the experimenter to mirror the path of the animal directly by placing the tablet's puck over the animal's image on the television screen and moving the puck as the animal swam. Using a glass tablet might increase, to some degree, the accuracy with which data were collected. But the greater cost of a glass tablet (approximately 3 to 5 times as much as an opaque tablet) and the somewhat lower likelihood of driver compatibility might limit this option's utility.

Although it is feasible for one person to collect data, the process is made easier with two. In the two-person variant, one person is responsible for placing the animal in the pool and running the stopwatch while the other performs digitizing duties. I have used the two-person procedure frequently, and it has worked well, especially with experimentally inexperienced students. In addition, the two-person procedure not only fosters a sense of collaboration, but also eliminates the very small potential for inaccurate data collection due to the few seconds ( 2 or 3) that elapse between placing the animal in the pool and returning to the digitizer. During this time, the lone experimenter must view the animal either directly in the pool or on the television screen, and then, upon arriving at the tablet, must digitize the swim path missed.

Besides assessing Morris maze performance, the computer-aided procedure described here can also be employed for collecting data in other behavioral research or instructional paradigms. Assessing open-field behavior, measuring stride lengths, determining how far an infant animal wanders from its mother, gauging the distances between food caches, and defining marked territories of individual animals are all examples of measurements that can be obtained with the present methodology. Moreover, the present procedure's utility is not limited to tests involving animal behavior. Assessing human behavior, such as children at play, interpersonal space, eye movements, and social interactions, can, for example, be similarly facilitated.

\section{Discussion}

An affordable and straightforward method for testing rodents in the Morris water maze has been described. Relative to commercially available computerized video tracking systems, the cost of the equipment is minimal (approximately $\$ 1,500$ for the video camera, tripod, television, computer software, and digitizing tablet), thus increasing the likelihood that research and instructional laboratories with small equipment budgets can afford to adopt such a procedure. In addition, the software does not require substantial system resources. In fact, although it is preferable to use a computer with a 386 or better processor, the software will work with a machine using an 8088 processor with $512 \mathrm{~K}$ of RAM and a monochrome monitor. (It should be noted that some configurations may require $640 \mathrm{~K}$ and that the GRAPHICS.COM DOS utility will not work with a monochrome graphics display directly.) In addition, although hard disk storage requirements are minimal, the software is compatible with at least one hard disk compres- 
sion routine (XtraDrive, Integrated Information Technology, Santa Clara, CA).

Apart from the cost, the attractiveness of this procedure is that undergraduate students learning experimental psychology methods, as well as experienced research assistants, can use the system with ease after only a relatively small amount of instruction. Although the present procedure is admittedly not as sophisticated or flexible as commercially available computerized video tracking systems, it does provide a reliable, affordable, and reasonable alternative.

\section{REFERENCES}

Denenberg, V. H., Talgo, N. W., Waters, N. S., \& Kenner, G. H. (1990). A computer-aided procedure for measuring Morris maze performance. Physiology \& Behavior, 47, 1027-1029.
Morris, R. G. M. (1981). Spatial location does not require the presence of local cues. Learning \& Motivation, 12, 239-260.

\section{NOTE}

1. Given an average swim rate of $24 \mathrm{~cm} / \mathrm{sec}$, it is estimated that distances for a $3-\mathrm{sec}$ and a $10-\mathrm{sec}$ trial would be 72 and $240 \mathrm{~cm}$, respectively. Applying the 11.1 standard deviation $(S)$ value of Rater 2 (i.e., the inexperienced rater), even if there were three standard deviation units of variability when measuring, the digitized distances for a $3-\mathrm{sec}$ and a $10-\mathrm{sec}$ trial would be $105.3(72+3 S)$ and 206.7 $(240-3 S) \mathrm{cm}$, respectively. In fact, given the same set of assumptions, digitized distances would only begin to overlap when a 3 -sec and a $5.7-\mathrm{sec}$ trial were compared $(105.3$ vs. $103.5 \mathrm{~cm}$, respectively).

(Manuscript received March 23, 1994; revision accepted for publication July 11,1994 .) 